\title{
Article \\ Generalized Guidance Formulation for Impact Angle Interception with Physical Constraints
}

\author{
Hyeong-Geun Kim ${ }^{1}\left(\mathbb{D}\right.$ and Jun-Yong Lee ${ }^{2, *(\mathbb{D})}$ \\ 1 Department of Mechanical Engineering, Incheon National University, Incheon 22012, Korea; hgkim@inu.ac.kr \\ 2 NearthLab, Seoul 06246, Korea \\ * Correspondence: junyong.lee@nearthlab.com; Tel.: +82-2-566-1574
}

Citation: Kim, H.-G.; Lee, J.-Y. Generalized Guidance Formulation for Impact Angle Interception with Physical Constraints. Aerospace 2021, 8, 307. https://doi.org/10.3390/ aerospace 8100307

Academic Editor: Gokhan Inalhan

Received: 6 September 2021

Accepted: 13 October 2021

Published: 18 October 2021

Publisher's Note: MDPI stays neutral with regard to jurisdictional claims in published maps and institutional affiliations.

Copyright: (C) 2021 by the authors. Licensee MDPI, Basel, Switzerland. This article is an open access article distributed under the terms and conditions of the Creative Commons Attribution (CC BY) license (https:// creativecommons.org/licenses/by/ $4.0 /)$.

\begin{abstract}
This paper proposes an optimal impact angle control guidance law for homing missiles with a narrow field-of-view of the seekers. As groundwork for designing a guidance law, we first present a general guidance structure that can achieve any terminal constraint of the line-of-sight rate based on the optimal control theory. We configure the desired profile of the line-of-sight rate using a saturation function whose exact form is determined to satisfy the required boundary conditions. By combining the line-of-sight rate profile with the optimal guidance structure, we develop a guidance law that achieves an impact angle interception with the field-of-view constraint. Herein, as the entire guidance structure is derived based on exact kinematics without any approximation, the proposed law ensures the accurate impact angle interception for various engagement scenarios. This precise consideration of the engagement kinematics also accurately ensures the energy optimality of preventing the excessive use of control inputs when homing. To evaluate the performance of the proposed method, numerical simulations with various engagement scenarios are conducted, and the results demonstrate that the proposed law allows missiles to accurately intercept their targets with the desired impact angles and without violating the prescribed field-of-view constraint.
\end{abstract}

Keywords: field-of-view limitation; impact angle control; nonlinear guidance; optimal guidance

\section{Introduction}

Proportional navigation guidance (PNG) has been widely used because it is known as an optimal method for minimizing flight energy and the terminal miss distances to stationary targets [1]. However, simple PNG does not ensure satisfactory performance if a target is armored or has a missile defense system such as a closed-in weapon system (CIWS) or an electronic countermeasure (ECM). To intercept targets by incapacitating these defense systems, guidance laws with additional capabilities, such as imposing terminal impact angles or impact times, are investigated. Particularly, impact angle control guidance (IACG) is one of the useful methods of effectively destroying the weak points on targets, and various approaches have been proposed to fulfill this capability.

In [2], an impact attitude angle control guidance law was proposed for re-entry vehicles using the linear quadratic control problem. In [3], a biased PNG was considered to intercept a moving maneuverable target. The capability of the guidance law with the desired impact angle was evaluated using the Lyapunov stability theory. A generalized form of the optimal guidance law considering the terminal impact angle constraint was studied in [4], while providing a method for estimating the corresponding time-to-go. In [5], as an improved work of [4], an optimal impact angle control problem was formulated based on the performance index that is inversely weighted by the time-to-go. The study in [6] suggests a guidance law that can control both the impact angle and time for a simultaneous attack of multiple missiles, and a guidance law for the same purpose was proposed by [7]. In this work, the shaping of the line-of-sight (LOS) and the second-order sliding mode approach were applied to satisfy the impact angle and time constraints. The authors of [8] 
and [9] investigated composite guidance laws that adjust the navigation gain of PNG to satisfy the impact angle constraint. A biased PNG form was used to develop the IACG law in [10] using the exact solution of nonlinear engagement dynamics. A polynomial structure that ensures the convergence of the command was used to configure the IACG law in [11], which was extended to a generalized formulation that provides solutions for weighted optimal impact angle control problems in [12]. The authors of [13-17] adopted nonlinear control techniques to achieve impact angle interception against a maneuvering target based on a kinematic formulation that ensures a high level of capturability.

In addition to the above-mentioned studies, in recent years, a lot of research has focused on the practical applications of IACG. For the homing missiles equipped with on-board seeker systems, in particular, the field-of-view (FOV) limitation is one of the most significant considerations, as IACG can cause a target to deviate from the seeker's FOV due to the requirement of a curved trajectory. The work presented in [18] is one of the pioneering studies that imposed the FOV constraint in the IACG problem. Based on the linearization of engagement kinematics, the optimal control with the inequality constraint of the specific state variable was used to configure the guidance law. The authors of [19] applied a similar approach with the use of a range-to-go weighted cost function. The inversely weighted range-to-go made the developed law generate the command converging to zero at the end of homing.

Dissimilar to the work in $[18,19]$, based on linearized engagement kinematics, the studies in [20-27] used exact nonlinear kinematics to design IACG laws with the FOV constraint. The authors of $[20,21]$ structured guidance laws as a composite PNG that switches the navigation gain at a specific instant. By appropriately adjusting the transition instant, both laws could achieve an impact angle interception without violating the prescribed FOV limit. The authors of $[22,23]$ investigated nonlinear kinematic conditions that ensure IACG with the FOV constraint, and guidance laws are designed to satisfy these conditions by only using the information of bearing angles. The authors of $[24,25]$ used the hyperbolic tangent function to configure a nonlinear guidance structure that ensures the fulfillment of IACG without violating the prescribed FOV constraint. The authors of [26] obtained an analytical solution that satisfies IACG, as well as the arrival time at the target, to design a guidance law that achieves an impact angle and time interception against a stationary target. The authors of [27] designed a guidance command as a biased PNG form that makes the look angle belong to a positively invariant set for the prescribed FOV limit. The presented nonlinear formulation also guarantees the convergence of the impact angle error before interception.

The nonlinear guidance laws presented in [20-27] ensure the accurate fulfillment of the required tasks owing to the consideration of precise engagement kinematics, which is not achievable by linear approaches. However, these existing nonlinear IACG laws can lead to an excessive use of control inputs, since they are not designed with the optimality of the input usage, unlike the linear optimal guidance laws presented in [18,19]. To guarantee the accurate satisfaction of desired tasks with the optimal use of control inputs, the development of an optimal guidance law that fulfills IACG with the FOV constraint based on exact nonlinear dynamics is required.

In this paper, we propose an optimal control-based guidance law considering the FOV constraint for the practical implementation of IACG. To configure the guidance law, we design an LOS rate profile based on which the desired tasks can be achieved using a saturation function whose magnitude is limited. In addition, the optimal control theory is used to derive the acceleration command that makes the actual LOS rate converge to the desired profile before interception. All the design processes are based on exact dynamics without any linear approximation; thus, enabling the proposed law to accurately satisfy the desired tasks as well as the optimality of the input usage.

In comparison with the previous studies that considered the IACG problem, our work has the following contributions. First, the proposed IACG law has the ability to prevent the look angle from exceeding prescribed limits. This allows missiles with seekers of a narrow 
FOV, such as strapdown seekers, to effectively conduct IACG, unlike the previous studies presented in [2-17].

Second, in this study, an optimal frame of the impact angle interception is built based on nonlinear kinematics without any linear approximation. Thus, the proposed law can ensure the accurate fulfillment of required tasks while preventing the excessive use of control inputs.

In addition, the proposed law does not require any iterative computation in the implementation, such as numerical optimization or the Newton-Raphson method. This eliminates the need to deal with the issue of performance reduction, which is caused by the lack of computation capacity or local minima. No existing studies that achieve all the above-stated contributions can be found in open literature despite the significance.

The rest of this paper is organized as follows: In Section 2, a framework for setting the engagement problem of IACG is formulated. In Section 3, a generalized guidance structure that achieves any given terminal constraint of the LOS rate is presented based on the optimal control theory. In Section 4, the LOS rate profile that satisfies the required boundary constraints is formulated to design the IACG law. In Section 5, the performance of the proposed law is evaluated through numerical simulations. Finally, concluding remarks are presented in Section 6.

\section{Problem Formulation}

Consider a planar geometry of engagement against a stationary target in the inertial coordinate frame $X_{I} O_{I} Y_{I}$, as shown in Figure 1. $r$ and $\lambda$ in the figure represent the relative range and line-of-sight (LOS) angle between the missile and target, which are denoted by $M$ and $T$, respectively, and $V_{M}, a_{M}$, and $\gamma_{M}$ represent the speed, normal acceleration, and flight path angle of the missile, respectively. In addition, $\sigma_{M}$ is the included angle between the missile velocity vector and the LOS, and it denotes the look angle with the assumption of a negligible angle-of-attack. Then, the governing equations of the engagement kinematics are given by:

$$
\begin{aligned}
\dot{r} & =-V_{M} \cos \sigma_{M} \\
r \dot{\lambda} & =-V_{M} \sin \sigma_{M} \\
\dot{\sigma}_{M} & =\frac{a_{M}}{V_{M}}-\frac{V_{\lambda}}{r}
\end{aligned}
$$

where $V_{\lambda}$ represents the relative component of the missile velocity normal to the LOS and is defined as $V_{\lambda}=r \dot{\lambda}$.

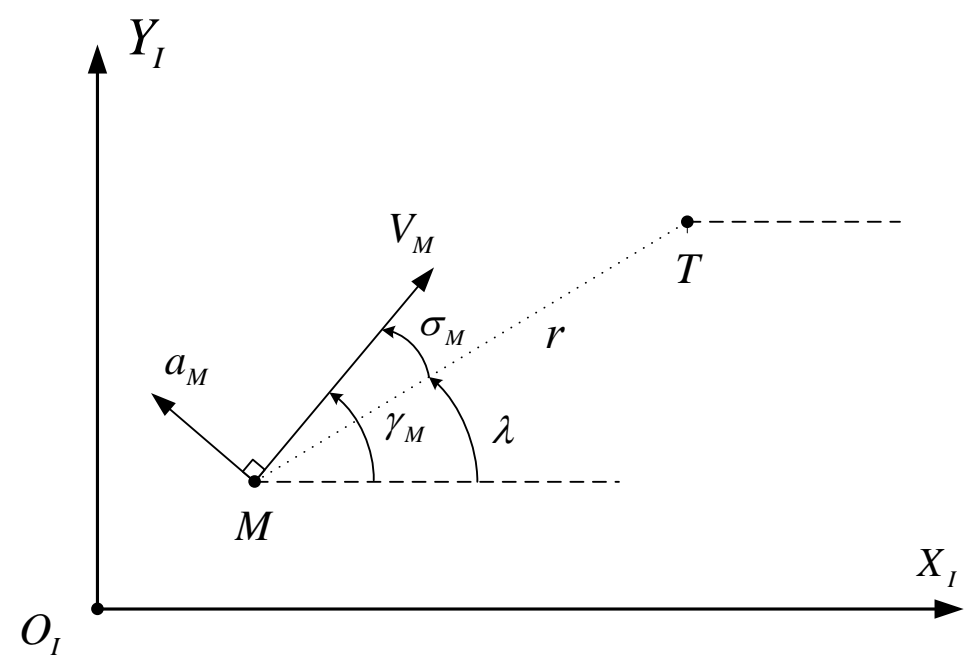

Figure 1. Engagement geometry for a stationary target. 
The objective of the proposed law is to intercept the target at the designated impact angle, while keeping the look angle from violating the prescribed limit. The mathematical expressions corresponding to the goals are as follows:

$$
\begin{aligned}
r\left(t_{f}\right) & =0 \\
\gamma_{M}\left(t_{f}\right) & =\gamma_{d} \\
\left|\sigma_{M}(t)\right| & \leq \sigma_{M}^{\max }<\pi / 2 \quad \forall t \in\left[0, t_{f}\right]
\end{aligned}
$$

where $t_{f}, \gamma_{d}$, and $\sigma_{M}^{\max }$ denote the final time, designated impact angle, and maximum limit of the look angle to consider the FOV constraint, respectively.

Note that the relative range $r$ eventually decreases to zero in common guidance scenarios as shown in (2a). Hence, as in the previous studies of $[18,19,28,29]$, we used (1a) to replace the time variable $t$ with the relative range $r$ as a differential variable as follows:

$$
\begin{aligned}
& \frac{d \lambda}{d r}=\frac{\tan \sigma_{M}}{r} \\
& \frac{d \dot{\lambda}}{d r}=\frac{a_{M}}{V_{M} r}-2 \frac{\dot{\lambda}}{r}
\end{aligned}
$$

At the instant of intercepting the target, the missile velocity vector and LOS must be in the same direction. Thus, the boundary conditions for the redefined dynamics in (3) are given by:

$$
\begin{gathered}
\left.\lambda(r)\right|_{r=0}=\gamma_{d} \\
\left.r \dot{\lambda}(r)\right|_{r=0}=0
\end{gathered}
$$

where the original boundary conditions in (2a) and (2b) were used. The FOV constraint in $(2 \mathrm{c})$ can also be rewritten as follows:

$$
\left|\sigma_{M}(r)\right| \leq \sigma_{M}^{\max }<\pi / 2 \quad \forall r \geq 0
$$

In this study, the guidance law was designed to satisfy the conditions of $(4 a) \sim(4 c)$ based on the dynamics of (3).

\section{Optimal Guidance Formulation to Achieve the Desired Constraints}

In this section, as groundwork for designing an IACG law, we presented a generalized guidance law that can achieve any given terminal constraint of the LOS rate based on the optimal control theory. The magnitude of the look angle under the presented guidance law was also analyzed to consider the seeker's FOV constraint.

Based on the dynamics of (3b), consider a guidance problem that requires the LOS rate to satisfy a given profile denoted by $\dot{\lambda}_{d}(r)$ at the end of homing. To achieve this requirement, we configured the guidance command as follows:

$$
a_{M}=a_{M}^{o p t}+2 V_{M} \dot{\lambda}_{d}+V_{M} r \frac{d \dot{\lambda}_{d}}{d r}
$$

where $a_{M}^{o p t}$ is the feedback control term whose quadratic summation is to be minimized by the optimal control theory. By substituting the command configuration of (5) into (3b), we obtained:

$$
\frac{d e_{\dot{\lambda}}}{d r}=\frac{a_{M}^{o p t}}{V_{M} r}-2 \frac{e_{\dot{\lambda}}}{r}
$$


where $e_{\dot{\lambda}}$ is the LOS rate error defined as $e_{\dot{\lambda}}=\dot{\lambda}-\dot{\lambda}_{d}$. Then, the problem of achieving $\dot{\lambda}_{d}$ was simplified to the regulation problem for $e_{\lambda}$ based on (6).

Now, to derive the optimal solution that achieves the regulation of $e_{\lambda}$, we presented the proposition below.

Proposition 1. Consider a quadratic performance index of:

$$
J\left(r_{0}\right)=\int_{0}^{r_{0}} \frac{1}{2 r^{m}} u^{2}(r) d r
$$

where $r_{0}, m$, and $u(r)$ are the initial value of the relative range, guidance gain (selected as a positive constant), and feedback control input (defined as $\left.u(r)=a_{M}^{o p t}(r) / V_{M}\right)$, respectively. Then, the optimal solution to minimize (7) subject to the dynamic constraint of (6) and the desired boundary condition of $e_{\dot{\lambda}}(0)=0$ is:

$$
a_{M}^{o p t}(r)=(m+3) V_{M} e_{\lambda}\left(r_{0}\right)\left(\frac{r}{r_{0}}\right)^{m+1}
$$

where $e_{\dot{\lambda}}\left(r_{0}\right)$ is the initial value of the LOS rate error defined as $e_{\dot{\lambda}}\left(r_{0}\right)=\dot{\lambda}_{0}-\dot{\lambda}_{d}\left(r_{0}\right)$ for the initial value of the LOS rate $\dot{\lambda}_{0}$.

Proof. The Hamiltonian corresponding to the performance index in (7) and the constraint in (6) is given by:

$$
\mathcal{H}=\frac{1}{2 r^{m}} u^{2}+v_{e}\left(\frac{u}{r}-2 \frac{e_{\dot{\lambda}}}{r}\right)
$$

where $v_{e}$ is the costate variable governed by:

$$
\frac{d v_{e}}{d r}=-\frac{\partial \mathcal{H}}{\partial e_{\lambda}}=2 \frac{v_{e}}{r}
$$

By solving (10), we obtained $v_{e}=c_{e} r^{2}$, where $c_{e}$ is the constant to be determined by the boundary condition. The optimality condition from (9) is given by:

$$
\frac{\partial \mathcal{H}}{\partial u}=0 \quad \Leftrightarrow \quad u=-c_{e} r^{m+1}
$$

Substituting (11) into (6) led to the first-order differential equation of:

$$
\begin{aligned}
& \frac{d e_{\dot{\lambda}}}{d r}=-c_{e} r^{m}-2 \frac{e_{\dot{\lambda}}}{r} \\
\Leftrightarrow & \frac{1}{r^{2}} \frac{d}{d r}\left(r^{2} e_{\dot{\lambda}}\right)=-c_{e} r^{m} \\
\Leftrightarrow & e_{\dot{\lambda}}=-\frac{c_{e}}{m+3} r^{m+1}
\end{aligned}
$$

where the boundary condition of $e_{\dot{\lambda}}(0)=0$ at $r=0$ was used. By using the other boundary condition of $e_{\lambda}\left(r_{0}\right)$ at $r=r_{0}$, we could determine the value of $c_{e}$ as $c_{e}=$ $-(m+3) e_{\dot{\lambda}}\left(r_{0}\right) / r_{0}^{m+1}$. Therefore, the optimal control input was derived as:

$$
\begin{aligned}
a_{M}^{\text {opt }}(r) & =V_{M} u(r) \\
& =(m+3) V_{M} e_{\lambda}\left(r_{0}\right)\left(\frac{r}{r_{0}}\right)^{m+1}
\end{aligned}
$$

which completes the proof. 
Using the result of Proposition 1, we had the guidance command that achieves $\dot{\lambda}=\dot{\lambda}_{d}$ at the end of homing as follows:

$$
a_{M}=(m+3) V_{M} e_{\dot{\lambda}}\left(r_{0}\right)\left(\frac{r}{r_{0}}\right)^{m+1}+2 V_{M} \dot{\lambda}_{d}+V_{M} r \frac{d \dot{\lambda}_{d}}{d r}
$$

Remark 1. For robust performance in actual implementation, it was necessary to configure the guidance command in the form of closed-loop feedback. To this end, we could use the current state variables rather than their initial values as boundary conditions to calculate the coefficient $c_{e}$ in (12) each time. Then, we obtained the real-time feedback command as follows:

$$
a_{M}=(m+3) V_{M} \dot{\lambda}-(m+1) V_{M} \dot{\lambda}_{d}+V_{M} r \frac{d \dot{\lambda}_{d}}{d r}
$$

The feedback form in (15) also shows that substituting $m=0$ and $\dot{\lambda}_{d}=0$ yields the wellknown PNG form. This result is consistent with the fact that PNG, with a navigation constant of three, is the optimal solution for intercepting a stationary target [1].

In addition to satisfying the terminal constraint, the FOV constraint could also be considered by appropriately selecting the desired profile of $\dot{\lambda}_{d}$. Further specifics are described in Proposition 2 below.

Proposition 2. Suppose that the desired profile of $\dot{\lambda}_{d}$ is selected to satisfy:

$$
\left|\frac{r \dot{\lambda}_{d}(r)}{V_{M}}\right| \leq \sin \sigma_{M}^{\max } \quad \forall r \geq 0
$$

and

$$
\frac{d}{d r}\left(r \dot{\lambda}_{d}(r)\right) \cdot \sigma_{M}(r) \leq 0 \quad \forall r \geq 0
$$

where $\sigma_{M}(r)$ in (17) is determined by the dynamics in (3) and the guidance command in (15). Then, the guidance command in (15) makes the set of $\left|\sigma_{M}(r)\right| \leq \sigma_{M}^{\max }$ an invariant set for all $r \geq 0$.

Proof. Using (1a) and (1c), we obtained the dynamics of the look angle with respect to the relative range as follows:

$$
\frac{d \sin \sigma_{M}}{d r}=-\frac{a_{M}}{V_{M}^{2}}-\frac{\sin \sigma_{M}}{r}
$$

Substituting (15) into (18) gave:

$$
\frac{d \sin \sigma_{M}}{d r}=\frac{(m+2)}{r}\left(\sin \sigma_{M}+\frac{r}{V_{M}} \dot{\lambda}_{d}\right)-\frac{1}{V_{M}} \frac{d}{d r}\left(r \dot{\lambda}_{d}\right)
$$

When $r \in \mathbb{B} \triangleq\left\{r \mid r \geq 0\right.$ and $\left.\sigma_{M}(r)=\sigma_{M}^{\max }\right\}$, the terms in (19) satisfied:

$$
\begin{aligned}
\left.\left(\sin \sigma_{M}+\frac{r}{V_{M}} \dot{\lambda}_{d}\right)\right|_{r \in \mathbb{B}} & =\sin \sigma_{M}^{\max }+\left.\frac{r}{V_{M}} \dot{\lambda}_{d}\right|_{r \in \mathbb{B}} \\
\geq & \sin \sigma_{M}^{\max }-\left|\frac{r \dot{\lambda}_{d}}{V_{M}}\right|_{r \in \mathbb{B}} \geq 0
\end{aligned}
$$


and

$$
\begin{aligned}
\left.\frac{d}{d r}\left(r \dot{\lambda}_{d}\right)\right|_{r \in \mathbb{B}} & =\left.\frac{d}{d r}\left(r \dot{\lambda}_{d}\right) \cdot \sigma_{M}(r)\right|_{r \in \mathbb{B}} \cdot \frac{1}{\sigma_{M}^{\max }} \\
& \leq 0
\end{aligned}
$$

where the conditions in (16) and (17) were used, respectively. Applying the properties in (20) and (21) into (19), we obtained:

$$
\left.\frac{d \sin \sigma_{M}}{d r}\right|_{\sigma_{M}=\sigma_{M}^{\max }} \geq 0 .
$$

which provided:

$$
\begin{aligned}
\left.\frac{d \sigma_{M}}{d t}\right|_{\sigma_{M}=\sigma_{M}^{\max }} & =-\left.V_{M} \frac{d \sin \sigma_{M}}{d r}\right|_{\sigma_{M}=\sigma_{M}^{\max }} \\
& \leq 0
\end{aligned}
$$

The result in (23) implies that the look angle $\sigma_{M}$ cannot exceed $\sigma_{M}^{\max }$ if the initial value starts within $\left[-\sigma_{M}^{\max }, \sigma_{M}^{\max }\right]$.

Propositions 1 and 2 showed that the proposed guidance formulation in (15) could satisfy any given terminal constraint of the LOS rate, as well as the FOV constraint if the conditions in (16) and (17) were achieved. Hence, by designing an appropriate profile of the LOS rate $\dot{\lambda}_{d}$, we could complete the design of the IACG law that achieves all requirements.

\section{Design of a Guidance Law for Impact Angle Interception with the FOV Constraint}

In this Section, we developed an IACG law using the optimal guidance formulation that was configured in Section 3. First, an LOS rate profile that ensures IACG without exceeding the prespecified limit was formulated as the groundwork. Next, we applied the profile to the generalized formulation in (15) to design the guidance law.

The impact angle interception was achieved if the LOS between the missile and target was maintained in the direction of the desired impact angle at the interception as shown in (4a) and (4b). In addition, if the magnitude of the look angle was restricted as in (4c), the FOV constraint could also be satisfied. To achieve all the requirements, we formulated the desired profile of the LOS rate as follows:

$$
\begin{aligned}
\dot{\lambda}_{d} & =-\frac{V_{M}}{r} \sin \left(\sigma_{M}^{\max } \operatorname{sat}\left(\frac{n}{\sigma_{M}^{\max }} e_{\lambda}\right)\right) \\
& = \begin{cases}-\frac{V_{M}}{r} \sin \sigma_{M}^{\max } \cdot \operatorname{sgn}\left(e_{\lambda}\right) & \text { for }\left|e_{\lambda}\right|>\frac{\sigma_{M}^{\max }}{V_{M}} \\
-\frac{V_{M}}{r} \sin \left(n e_{\lambda}\right) & \text { for }\left|e_{\lambda}\right| \leq \frac{\sigma_{M}^{\max }}{n}\end{cases}
\end{aligned}
$$

where $e_{\lambda}, n$, and sat $(\cdot)$ represent the LOS error defined as $e_{\lambda}=\lambda-\gamma_{d}$, the guidance gain selected as a positive constant larger than or equal to 1 , and the continuous saturation function defined as $\operatorname{sat}(x)=x$ if $|x| \leq 1$ and $\operatorname{sat}(x)=\operatorname{sgn}(x)$ otherwise, respectively. Then, related to the IACG, the desired LOS rate in (24) led to the following proposition:

Proposition 3. Suppose that the dynamics of the LOS are governed by the desired LOS rate defined by (24). Then, the LOS angle $\lambda$ achieves the terminal constraints of (4a) and (4b) for any initial conditions. 
Proof. Under the premise of $\dot{\lambda}=\dot{\lambda}_{d}$, the LOS error $e_{\lambda}$ satisfied the dynamics of:

$$
\left.\frac{d e_{\lambda}}{d r}\right|_{\dot{\lambda}_{=} \dot{\lambda}_{d}}= \begin{cases}\operatorname{sgn}\left(e_{\lambda}\right) \cdot \frac{\tan \sigma_{M}^{\max }}{r} & \text { for }\left|e_{\lambda}\right|>\frac{\sigma_{M}^{\max }}{n} \\ \frac{\tan \left(n e_{\lambda}\right)}{r} & \text { for }\left|e_{\lambda}\right| \leq \frac{\sigma_{M}^{\max }}{n}\end{cases}
$$

where the LOS dynamics in (3a) was used. Integrating both sides of (25) yielded:

$$
\left.e_{\lambda}\right|_{\dot{\lambda}_{=} \dot{\lambda}_{d}}= \begin{cases}e_{\lambda}\left(r_{0}\right)+\operatorname{sgn}\left(e_{\lambda}\left(r_{0}\right)\right) \tan \sigma_{M}^{\max } \log \left(\frac{r}{r_{0}}\right) & \text { for } r>r_{1} \\ \frac{\operatorname{sgn}\left(e_{\lambda}\left(r_{1}\right)\right)}{n} \sin ^{-1}\left\{\sin \sigma_{M}^{\max }\left(\frac{r}{r_{1}}\right)^{n}\right\} & \text { for } r \leq r_{1}\end{cases}
$$

where $e_{\lambda}\left(r_{0}\right)$ and $r_{1}$ denote the initial value of the LOS error and the relative range at $\left|e_{\lambda}\left(r_{1}\right)\right|=\sigma_{M}^{\max } / n$, respectively. The closed-loop solution of $e_{\lambda}$ in (26) shows that $\lambda$ converges to $\gamma_{d}$ as $r$ goes to zero, regardless of the initial conditions. This proves (4a) under the assumption of $\dot{\lambda}=\dot{\lambda}_{d}$.

Using (26), we could also derive the closed-loop solution for $\dot{\lambda}$ as:

$$
\dot{\lambda}_{d}= \begin{cases}-\operatorname{sgn}\left(e_{\lambda}\left(r_{0}\right)\right) \frac{V_{M} \sin \sigma_{M}^{\max }}{r} & \text { for } r>r_{1} \\ -\operatorname{sgn}\left(e_{\lambda}\left(r_{1}\right)\right) \frac{V_{M} \sin \sigma_{M}^{\max }}{r_{1}}\left(\frac{r}{r_{1}}\right)^{n-1} & \text { for } r \leq r_{1}\end{cases}
$$

The result in (27) proves that (4b) under the assumption of $\dot{\lambda}=\dot{\lambda}_{d}$ as the gain $n$ is chosen to be larger than or equal to 1.

Proposition 3 implies that the impact angle interception, which is mathematically expressed as (4a) and (4b), can be executed if $\dot{\lambda}=\dot{\lambda}_{d}$ is achieved at any time during homing. Therefore, the selection of (24) as the desired profile of the LOS rate enabled the guidance law in (15) to fulfill IACG, owing to the capability to satisfy the pre-specified terminal constraint, as proven by Proposition 1.

Furthermore, the desired profile in (24) satisfied that:

$$
\begin{aligned}
\left|\frac{r \dot{\lambda}_{d}}{V_{M}}\right| & =\left|\sin \left(\sigma_{M}^{\max } \operatorname{sat}\left(\frac{n}{\sigma_{M}^{\max }} e_{\lambda}\right)\right)\right| \\
& \leq\left|\sin \sigma_{M}^{\max }\right|
\end{aligned}
$$

and

$$
\begin{aligned}
& \frac{d}{d r}\left(r \dot{\lambda}_{d}\right) \sigma_{M} \\
& \quad= \begin{cases}\frac{d}{d r}\left(\mp V_{M} \sin \sigma_{M}^{\max }\right) \cdot \sigma_{M} & \text { for }\left|e_{\lambda}\right|>\frac{\sigma_{M}^{\max }}{n} \\
\frac{d}{d r}\left(-V_{M} \sin \left(n e_{\lambda}\right)\right) \cdot \sigma_{M} & \text { for }\left|e_{\lambda}\right| \leq \frac{\sigma_{M}^{\max }}{n}\end{cases} \\
& \quad= \begin{cases}0 & \text { for }\left|e_{\lambda}\right|>\frac{\sigma_{M}^{\max }}{n} \\
-n V_{M} \cos \left(n e_{\lambda}\right) \frac{\tan \sigma_{M}}{r} \sigma_{M} & \text { for }\left|e_{\lambda}\right| \leq \frac{\sigma_{M}^{\max }}{n}\end{cases} \\
& \quad \leq 0
\end{aligned}
$$

where the dynamics of $\lambda$ with respect to $r$ in (3a) were used. The results in (28) and (29) indicate that the presented profile $\dot{\lambda}_{d}$ in (24) satisfied the conditions of (16) and (17) in Proposition 2. Hence, the proposed guidance law in (15) with the use of the profile in (24) prevents the look angle $\sigma_{M}$ from violating the prescribed limit $\sigma_{M}^{\max }$ and realizes the impact angle interception. 
Remark 2. Considering the situation in which there is no FOV constraint, the proposed guidance law only takes the case of $\left|e_{\lambda}\right| \leq \sigma_{M}^{\max } / n$ in (24). Then, the proposed law for the purpose of only IACG is given by:

$$
\begin{aligned}
& a_{M}=(m+3) V_{M} \dot{\lambda}-(m+2) V_{M} \dot{\lambda}_{d}+V_{M} \frac{d}{d r}\left(r \dot{\lambda}_{d}\right) \\
& =(m+3) V_{M} \dot{\lambda}-(m+2) V_{M} \dot{\lambda}_{d}-n V_{M}^{2} \cos \left(n e_{\lambda}\right) \frac{\tan \sigma_{M}}{r}
\end{aligned}
$$

In addition, applying a small-angle approximation to the trigonometric functions in (30) led to:

$$
\begin{aligned}
a_{M} \approx & \frac{V_{M}}{t_{g o}}\left\{(m+3)(n+1) \lambda-(m+n+3) \gamma_{M}\right. \\
& \left.-(m+2) n \gamma_{d}\right\}
\end{aligned}
$$

where the time-to-go $t_{g o}$ is approximated as $t_{g o} \approx r / V_{M}$. Herein, if $m$ and $n$ are selected as $m=N$ and $n=N+1$ for any non-negative constant $N$, the approximated command in (31) is identical to the time-to-go weighted optimal IACG law in [5]. This shows that the proposed guidance law could also be generalized to the optimal solution of the IACG problem, which does not involve the FOV constraint.

\section{Performance Demonstration through Numerical Simulation}

In this section, we investigated the performance of the proposed guidance law through two subsections. First, in Section 5.1, we analyzed the characteristics of the proposed law by performing simulations for various engagement scenarios. Next, in Section 5.2, we compared the proposed law with other FOV-constrained IACG laws to demonstrate the contributions of this study.

In all the simulations, we used the feedback form in (15) with the desired LOS rate in (24) as the guidance command. That is, the proposed law was set to generate the command in an expression of:

$$
a_{M}=\left\{\begin{array}{cl}
(m+3) V_{M} \dot{\lambda}-(m+2) V_{M} \dot{\lambda}_{d} & \text { for }\left|e_{\lambda}\right|>\frac{\sigma_{M}^{\max }}{n} \\
(m+3) V_{M} \dot{\lambda}-(m+2) V_{M} \dot{\lambda}_{d} & \text { for }\left|e_{\lambda}\right| \leq \frac{\sigma_{M}^{\max }}{n} \\
-n V_{M}^{2} \cos \left(n e_{\lambda}\right) \frac{\tan \sigma_{M}}{r} & \text {. }
\end{array}\right.
$$

In addition, considering autopilot dynamics, all the commands used in the simulations were applied with a time constant of $0.1 \mathrm{~s}$. The termination condition of every simulation is when the relative range is less than or equal to $0.1 \mathrm{~m}$.

\subsection{Performance Analysis of the Proposed Guidance Law}

Figures 2 and 3 illustrate the simulation results under various settings, given by Table 1, to evaluate the proposed law. First, Figure $2 \mathrm{a}-\mathrm{f}$ presents the results under various settings of the gains $m$ and $n$ with a fixed impact angle and FOV constraints of $\gamma_{d}=-90^{\circ}$ and $\sigma_{M}^{\max }=45^{\circ}$. The missile trajectories given by Figure 2a,d show that the proposed law could achieve the interception of the target with the prescribed impact angle of $-90^{\circ}$ for all cases. The specific impact angles under the settings of $(m, n)=(0,1),(1,1),(2,1)$, $(3,1),(0,2),(0,3)$, and $(0,4)$ were produced as $\gamma_{M}\left(t_{f}\right)=-89.999^{\circ},-89.999^{\circ},-90.000^{\circ}$, $-90.000^{\circ},-90.000^{\circ},-90.000^{\circ}$, and $-90.000^{\circ}$, respectively. These results indicate that the proposed law fulfilled IACG with an accuracy within $\left|\gamma_{M}\left(t_{f}\right)-\gamma_{d}\right| \leq 1 \times 10^{-3 \circ}$.

Figure $2 \mathrm{~b}$,e shows that the proposed law made the errors $e_{\lambda}$ and $e_{\dot{\lambda}}$ go to zero at the end of homing, as theoretically proven by Propositions 1 and 3. From the second row of Figure $2 \mathrm{~b}$, it can be seen that the error $e_{\dot{\lambda}}$ converged faster during the initial stage of homing as a larger $m$ was selected. This was due to the structure of the performance index 
used to derive the proposed optimal guidance law. As shown in (7), the minimization of the command during the initial stage of homing was less weighted as a larger $m$ was selected. That is, $m$ acted as a gain that concentrated on rapidly reducing $e_{\lambda}$ in the initial stage. On the contrary, the first row of Figure 2e shows that larger $n$ values made the terminal convergence rate of $e_{\lambda}$ faster. This result was consistent with the analytic solution for $e_{\lambda}$ in (26), where $n$ acted as an exponent for $r$ in the terminal convergence of $e_{\lambda}$.

The first rows of Figure $2 \mathrm{c}, \mathrm{f}$ demonstrate that the proposed law did not violate the prescribed FOV constraint of $\sigma_{M}^{\max }=45^{\circ}$ under all the considered gain settings. The acceleration histories in the second row of Figure 2c show that the acceleration command was more concentrated in the initial stage than in the terminal stage as a larger $m$ was selected, which could also be confirmed by the performance index design in (7).

Table 1. Simulation settings.

\begin{tabular}{cc}
\hline Parameters & Values \\
\hline Initial missile position $\left(x_{M}(0), y_{M}(0)\right)$ & $(0,0) \mathrm{km}$ \\
Stationary target position $\left(x_{T}, y_{T}\right)$ & $(10,0) \mathrm{km}$ \\
Initial missile flight path angle $\gamma_{M}(0)$ & $15^{\circ}$ \\
Missile speed $V_{M}$ & $250 \mathrm{~m} / \mathrm{s}$ \\
Look angle limit $\sigma_{M}^{\max }$ & $30^{\circ}, 45^{\circ}, 60^{\circ}$ \\
Desired impact angle $\gamma_{d}$ & $-30^{\circ},-60^{\circ},-90^{\circ},-120^{\circ}$ \\
Setting of gains $(m, n)$ & $(0,1),(1,1),(2,1),(3,1)$, \\
& $(0,2),(0,3),(0,4)$ \\
\hline
\end{tabular}

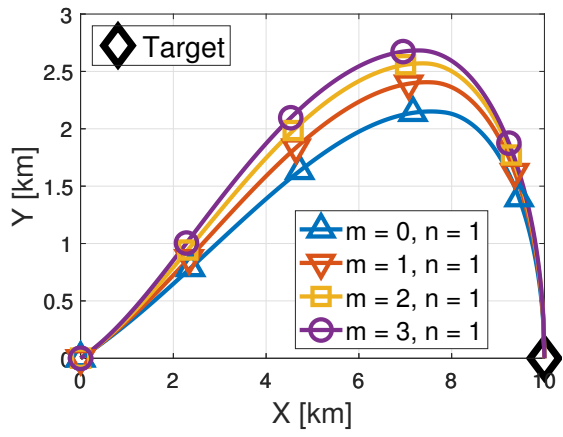

(a)

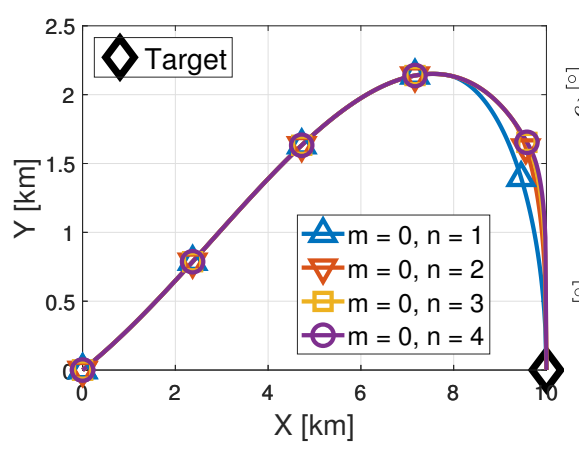

(d)
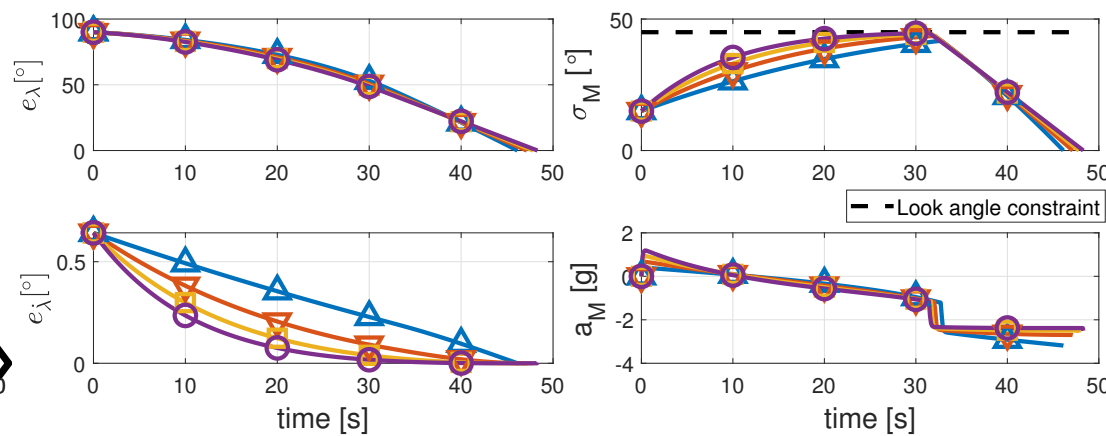

(b)

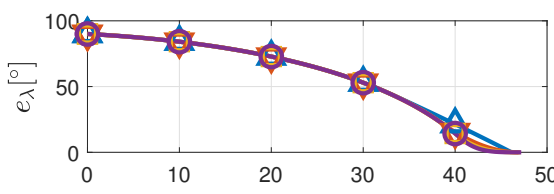

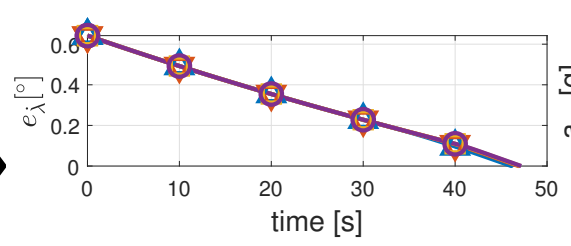

(e)
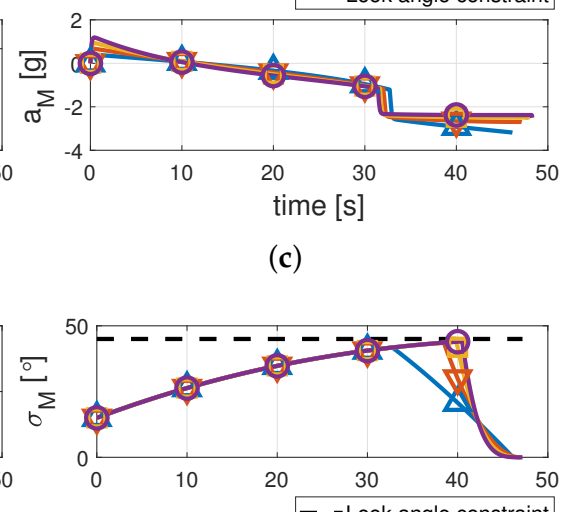

(c)

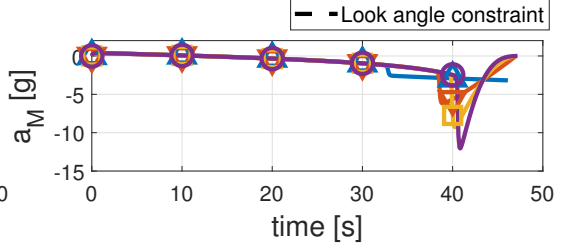

(f)

Figure 2. Simulation results of the proposed law under various setting of gains. (a) Flight trajectories. (b) Error variables. (c) Look angles and normal accelerations. (d) Flight trajectories. (e) Error variables. (f) Look angles and normal accelerations. 


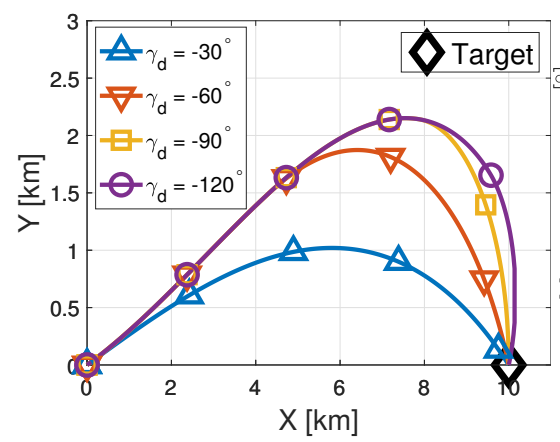

(a)

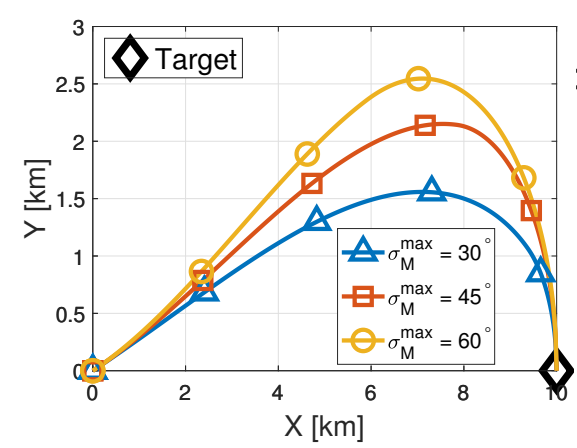

(d)
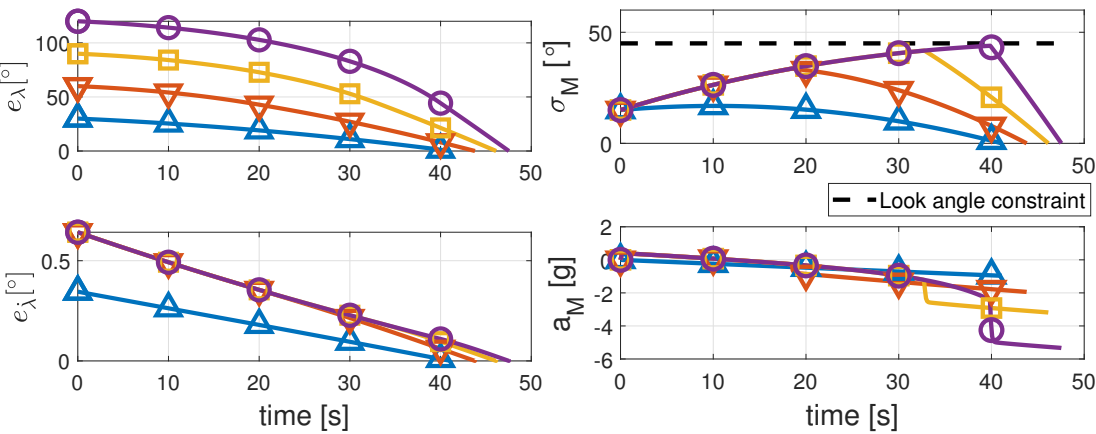

(b)

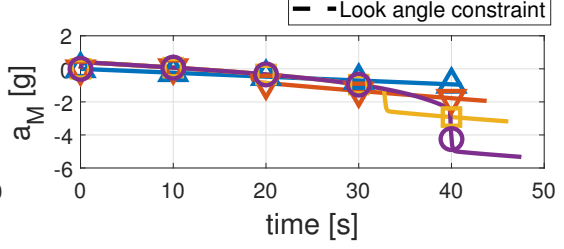

(c)
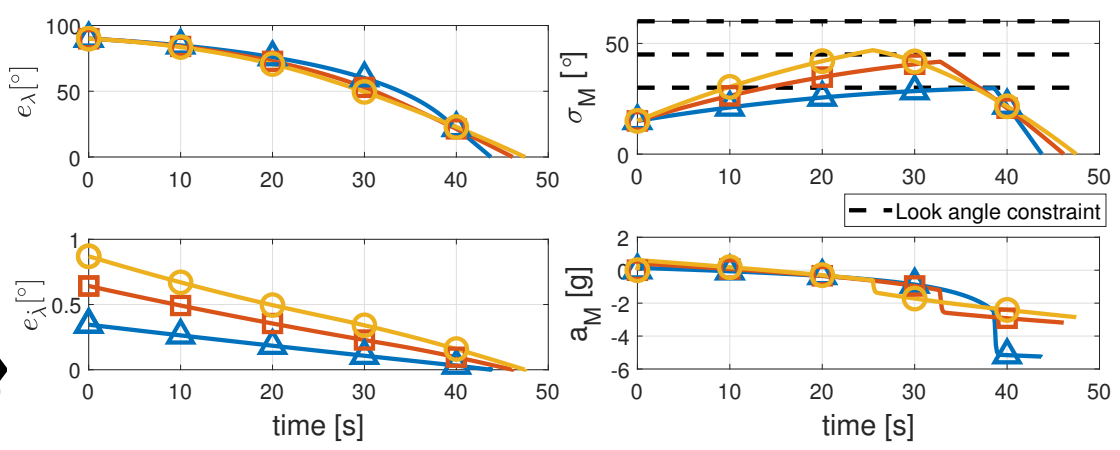

(e)

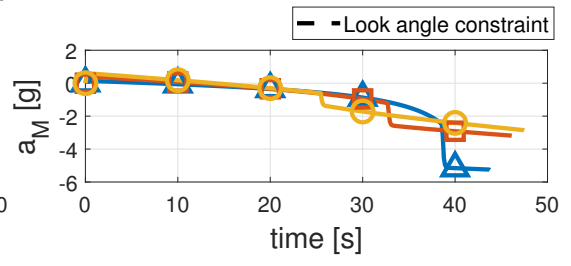

(f)

Figure 3. Simulation results of the proposed law for various impact angle and look angle constraints. (a) Flight trajectories. (b) Error variables. (c) Look angles and normal accelerations. (d) Flight trajectories. (e) Error variables. (f) Look angles and normal accelerations.

Figure $3 a-c$ provides the results of the desired impact angles of $\gamma_{d}=-30^{\circ},-60^{\circ}$, $-90^{\circ}$, and $-120^{\circ}$ with the maximum FOV constraint of $\sigma_{M}^{\max }=45^{\circ}$ under the fixed gains of $m=0$ and $n=1$. The proposed law performed the interception of the target with the impact angles of $-29.999^{\circ},-59.999^{\circ},-89.999^{\circ}$, and $-119.999^{\circ}$ in each scenario, which implied that the IACG was conducted with an accuracy within $\left|\gamma_{M}\left(t_{f}\right)-\gamma_{d}\right| \leq 1 \times 10^{-3 \circ}$.

Figure $3 \mathrm{c}$ shows that the proposed law kept the look angle from exceeding the limit of $\sigma_{M}^{\max }=45^{\circ}$ with the command within $-6 g \leq a_{M} \leq g$. It can also be seen that the maximum value of the guidance command tended to increase with the increase in the required impact angle because the missile must perform maneuvers within the limited FOV to perform IACG.

Figure 3d-f illustrates the scenarios in which the FOV constraints of $\sigma_{M}^{\max }=30^{\circ}, 45^{\circ}$, and $60^{\circ}$ were considered for the fixed impact angle of $-90^{\circ}$ and guidance gains of $m=0$ and $n=1$. Figure $3 \mathrm{~d}-\mathrm{f}$ shows that the proposed law fulfilled vertical attacks against the target without violating various FOV constraints. Specifically, the resulting impact angles satisfied accuracies within $\left|\gamma_{M}\left(t_{f}\right)-\gamma_{d}\right| \leq 1 \times 10^{-3 \circ}$ in all scenarios. Figure 3f also exhibits that the maximum value of the acceleration command tended to increase as the allowable FOV range was narrower. This was because the turning radius for performing IACG was shortened due to the narrow FOV constraint, which was similar to the case in Figure 3c.

\subsection{Performance Comparison with Other IACG Laws}

We compared the performances of the proposed guidance law and other IACG laws, range-to-go weighted optimal guidance law (ROG), and integral barrier Lyapunov functionbased guidance law (IBLG), which were presented in $[19,24]$, respectively. Both laws were designed to achieve an impact angle interception without violating the pre-specified FOV constraint against a stationary target. In particular, ROG can ensure optimal interceptions 
in linearized spaces, and IBLG guarantees the precise satisfaction of desired tasks based on exact nonlinear formulations. Both ROG and IBLG were used in this simulation through the following commands:

ROG [19]:

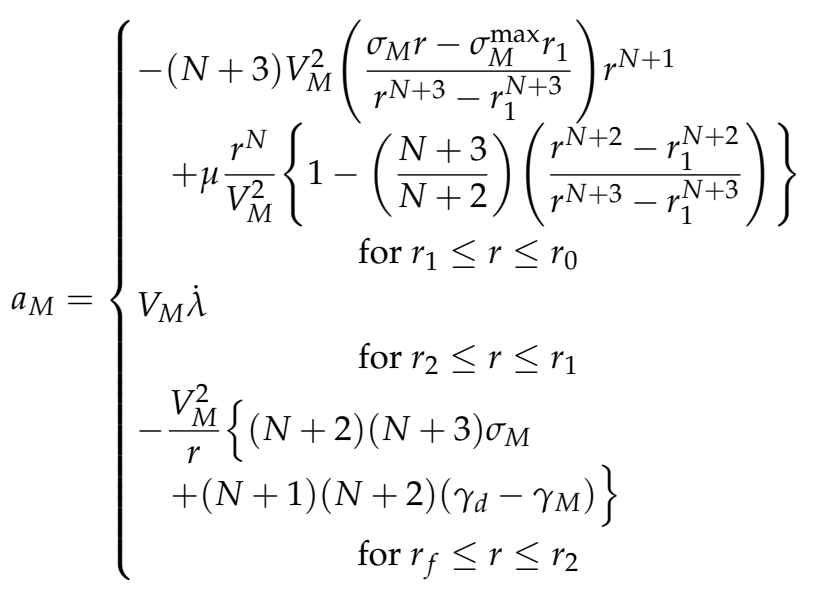

IBLG [24]:

$$
\begin{aligned}
a_{M}= & \frac{V_{M}\left(k_{b}^{2}-\sigma_{M}^{2}\right)}{k_{b}^{2}}\left(-c_{2} e_{2}+\frac{V_{M}}{r} f\left(\sigma_{M}\right) e_{1}+\chi\right) \\
& +V_{M}\left(\frac{k_{b}^{2}-\sigma_{M}^{2}}{k_{b}^{2}} \dot{\sigma}_{M}^{d} \rho\left(e_{2}, \sigma_{M}^{d}\right)+\dot{\lambda}\right)
\end{aligned}
$$

We set the gain of $N$ in (33) as $N=1$ to make the command converge to zero at the end of homing. For the same purpose, we set the gains of the proposed law as $m=1$ and $n=2$. The specific settings of the other parameters in (33) and (34) can be found in [19,24].

For a more reliable comparison from a practical point of view, we additionally introduced a time-varying speed model as follows:

$$
\dot{V}_{M}=\frac{T-D}{m}-g \sin \gamma_{M}
$$

where $T, D$, and $m$ denote the longitudinal main thrust, drag force, and time-varying mass of the missile modeled as:

$$
\begin{aligned}
T & = \begin{cases}33000 & 0 \leq t \leq 1.5 \\
7500 & 1.5 \leq t \leq 8.5 \\
0 & 8.5 \leq t\end{cases} \\
D & =C_{d 0} Q S_{r e f}+\frac{K_{i} m^{2} a_{M}^{2}}{Q S_{r e f}} \\
m & = \begin{cases}135-14.53 t & 0 \leq t \leq 1.5 \\
113.205-3.31 t & 1.5 \leq t \leq 8.5 \\
90.035 & 8.5 \leq t\end{cases}
\end{aligned}
$$

where $Q, S_{r e f}, C_{d 0}$, and $K_{i}$ in (37) denote the dynamic pressure, reference area, zero-lift drag coefficient, and induced drag coefficient, respectively, and their specific values can be found in [30]. The input saturation for the normal acceleration was also considered as $\left|a_{M}\right| \leq 10 \mathrm{~g}$, and all the guidance laws were set to achieve the desired impact angle of $-90^{\circ}$ under the FOV constraint of $\sigma_{M}^{\max }=45^{\circ}$.

Figure $4 \mathrm{a}-\mathrm{f}$ illustrates the simulation results of three guidance laws in ideal and realistic scenarios, respectively. The realistic scenario included a time-varying speed model 
in (35), while the ideal scenario used a constant speed model, as in Section 5.1. The specific histories of the missile speed in each scenario can be seen in the second rows of Figure $4 \mathrm{~b}$,e. It can be observed that the speed in the realistic scenario initially increased due to the thrust in (36) and then decreased due to the drag in (37).

Figure $4 a, b, d, e$, shows that all three guidance laws achieved vertical interception of the targets without violating the FOV constraint of $\sigma_{M}^{\max }=45^{\circ}$ in both scenarios. The resulting impact angles under all guidance laws were within $\left|\gamma_{M}\left(t_{f}\right)-\gamma_{d}\right| \leq 1 \times$ $10^{-30}$ in every case.

Figure $4 \mathrm{c}, \mathrm{f}$ presents time histories of the normal acceleration and quadratic sum $\int_{0}^{t} a_{M}^{2} d t$ in each scenario to investigate the input usage of each guidance law. Figure $4 c$ shows that ROG and the proposed law consumed less commands than IBLG in the ideal scenario owing to the consideration of optimality in the input usage. The terminal values of the total quadratic sums of ROG, IBLG, and the proposed law were computed as $\int_{0}^{t_{f}} a_{M}^{2} d t=1.504 \times 10^{4}, 2.127 \times 10^{4}$, and $1.394 \times 10^{4} \mathrm{~m}^{2} / \mathrm{s}^{3}$, respectively.

The realistic scenario in Figure $4 \mathrm{f}$ shows that the terminal values of the total quadratic sums of ROG, IBLG, and the proposed law were computed as $\int_{0}^{t_{f}} a_{M}^{2} d t=3.820 \times 10^{4}$, $3.572 \times 10^{4}$, and $1.707 \times 10^{4} \mathrm{~m}^{2} / \mathrm{s}^{3}$, respectively. Dissimilar to the ideal scenario, ROG required a larger amount of the command than the other two laws because of the need for a large command in the initial stage, as shown in the first row of Figure 4f. The main reason for not guaranteeing the optimality of the guidance command was that the switching instant of ROG was not determined in real-time according to uncertainties and disturbances. As can be confirmed in [19], the computation of the switching instant for ROG required a numerical iterative routine that is hard to perform in real-time, so it was difficult to expect optimality in the practical applications with various uncertainties.

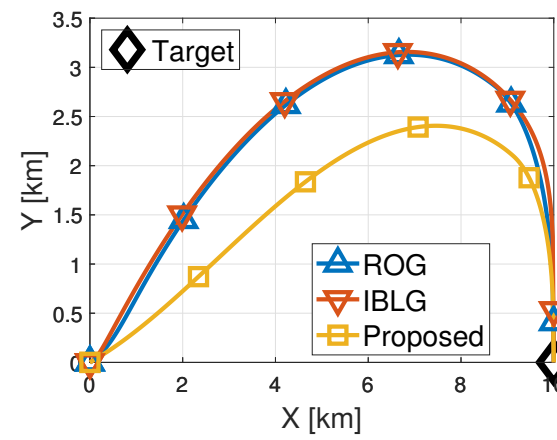

(a)

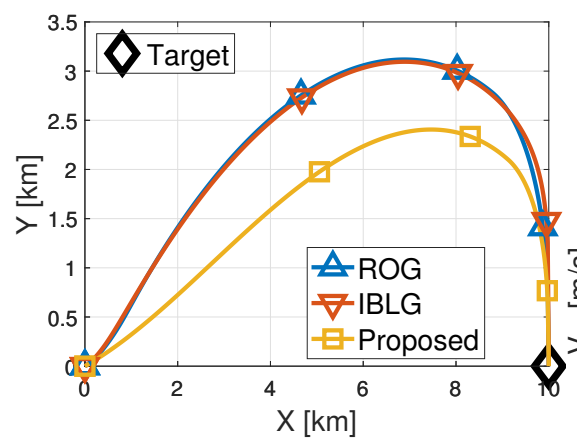

(d)

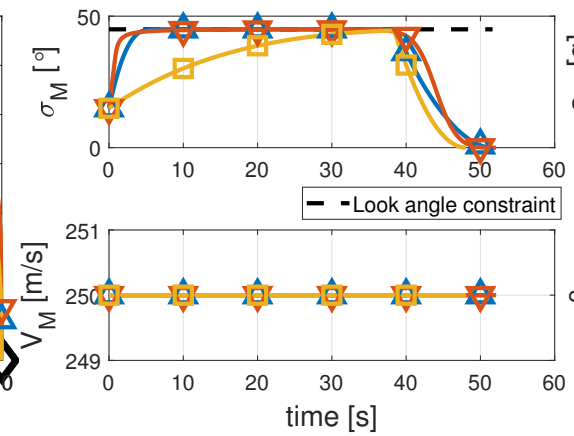

(b)

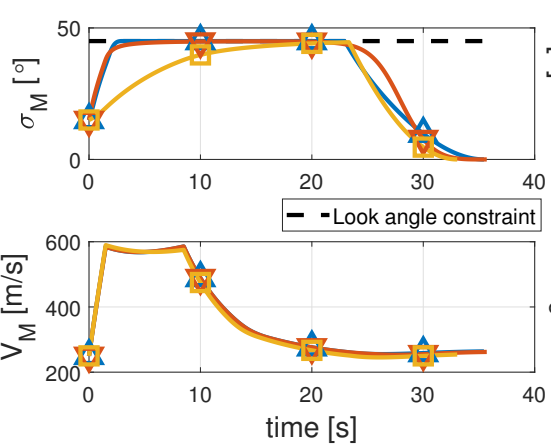

(e)

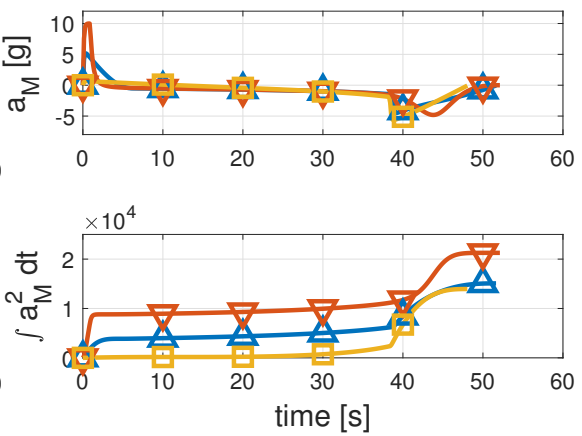

(c)
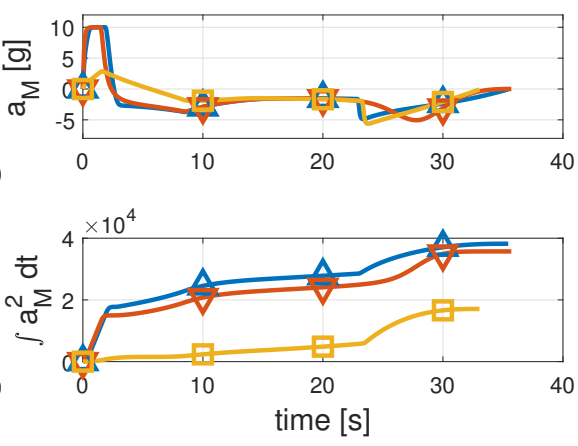

(f)

Figure 4. Simulation results of ROG, IBLG, and the proposed law in ideal (a-c) and realistic (d-f) scenarios. (a) Flight trajectories. (b) Look angles and speeds. (c) Normal accelerations and quadratic sums. (d) Flight trajectories. (e) Look angles and speeds. (f) Normal accelerations and quadratic sums. 
On the contrary, the proposed law produced an optimal command in real time without any numerical computations, as shown in (32), so it could retain characteristics close to optimal even under uncertainties. For such a reason, it was observed that the difference in the quadratic sums between each scenario was not large compared with the cases of ROG. That is, it was expected that the proposed law could perform IACG with less guidance command than the other two laws in actual implementations.

\section{Conclusions}

This study proposed a nonlinear guidance law that aimed at intercepting a stationary targets at designated impact angles without violating the maximum FOV constraint. The presented guidance law was configured as an optimal controller that achieves the desired LOS rate structured as a saturated feedback of the impact angle error. Since the satisfaction of the desired profile ensured the impact angle interception without violating the FOV constraint, the proposed law can satisfy all the required tasks. Dissimilar to most previous FOV-constrained IACG laws, the proposed law guarantees optimality in the engagement kinematics that do not involve any linearization, even without the use of a numerical iterative computation. Hence, the proposed law can ensure the accurate satisfaction of desired tasks, as well as prevent the excessive use of a guidance command. In addition, the optimal guidance structure proposed in this study had the capability of satisfying terminal constraints for any given LOS rate profile, so it can be applied to other guidance problems. Thus, this study could also be very useful in terms of versatility. The numerical simulations that were performed for the performance evaluation demonstrated that the proposed law precisely fulfilled the impact angle interception with less use of guidance commands compared with other existing IACG laws.

Author Contributions: Conceptualization, H.-G.K.; Validation, H.-G.K. and J.-Y.L.; Writing—original draft preparation, H.-G.K. and J.-Y.L.; Writing—review and editing, J.-Y.L. All authors have read and agreed to the published version of the manuscript.

Funding: This work was supported by the Incheon National University Research Grant in 2019.

Institutional Review Board Statement: Not applicable.

Informed Consent Statement: Not applicable.

Conflicts of Interest: The authors declare no conflict of interest.

\section{References}

1. Bryson, A.E.; Ho, Y.C. Applied Optimal Control; Hemisphere: Washington, DC, USA, 1975.

2. Kim, M.; Grider, K.V. Terminal guidance for impact attitude angle constrained flight trajectories. IEEE Trans. Aerosp. Electron. Syst. 1973, 9, 852-859. [CrossRef]

3. Kim, B.S.; Lee, J.G.; Han, H.S. Biased PNG law for impact with angular constraint. IEEE Trans. Aerosp. Electron. Syst. 1998, 34, 277-288.

4. Ryoo, C.K.; Cho, H.; Tahk, M.J. Optimal guidance laws with terminal impact angle constraint. J. Guid. Control Dyn. 2005, 28, 724-732. [CrossRef]

5. Ryoo, C.K.; Cho, H.; Tahk, M.J. Time-to-go weighted optimal guidance with impact angle constraints. IEEE Trans. Control Syst. Technol. 2006, 14, 483-492. [CrossRef]

6. Lee, J.I.; Jeon, I.S.; Tahk, M.J. Guidance law to control impact time and angle. IEEE Trans. Aerosp. Electron. Syst. 2007, 43, 301-310.

7. Harl, N.; Balakrishnan, S.N. Impact time and angle guidance with sliding mode control. IEEE Trans. Control Syst. Technol. 2012, 20, 1436-1449. [CrossRef]

8. Ratnoo, A.; Ghose, D. Impact angle constrained interception of stationary targets. J. Guid. Control Dyn. 2008, 31, 1816-1821. [CrossRef]

9. Ratnoo, A.; Ghose, D. Impact angle constrained guidance against nonstationary nonmaneuvering targets. J. Guid. Control Dyn. 2010, 33, 269-275. [CrossRef]

10. Akhil, G.; Ghose, D. Biased PN based impact angle constrained guidance using a nonlinear engagement model. In Proceedings of the American Control Conference (ACC), Montreal, QC, Canada, 27-29 June 2012; pp. 950-955.

11. Lee, C.H.; Kim, T.H.; Tahk, M.J.; Whang, I.H. Polynomial guidance laws considering terminal impact angle and acceleration constraints. IEEE Trans. Aerosp. Electron. Syst. 2013, 49, 74-92. [CrossRef] 
12. Lee, C.H.; Tahk, M.J.; Lee, J.I. Generalized formulation of weighted optimal guidance laws with impact angle constraint. IEEE Trans. Aerosp. Electron. Syst. 2013, 49, 1317-1322. [CrossRef]

13. Kumar, S.R.; Rao, S.; Ghose, D. Sliding-mode guidance and control for all-aspect interceptors with terminal angle constraints. J. Guid. Control Dyn. 2012, 35, 1230-1246. [CrossRef]

14. Rao, S.; Ghose, D. Terminal Impact Angle Constrained Guidance Laws Using Variable Structure Systems Theory. IEEE Trans. Control Syst. Technol. 2013, 21, 2350-2359. [CrossRef]

15. Kumar, S.R.; Rao, S.; Ghose, D. Nonsingular terminal sliding mode guidance with impact angle constraints. J. Guid. Control Dyn. 2014, 37, 1114-1130. [CrossRef]

16. Cho, D.; Kim, H.J.; Tahk, M.J. Impact angle constrained sliding mode guidance against maneuvering target with unknown acceleration. IEEE Trans. Aerosp. Electron. Syst. 2015, 51, 1310-1323. [CrossRef]

17. Kim, H.G.; Kim, H.J. All-aspect Guidance with Impact Angle Constraint against Unknown Target Maneuver. IEEE Trans. Aerosp. Electron. Syst. 2019, 55, 830-845. [CrossRef]

18. Park, B.G.; Kim, T.H.; Tahk, M.J. Optimal impact angle control guidance law considering the seeker's field-of-view limits. Proc. Inst. Mech. Eng. Part G J. Aerosp. Eng. 2013, 227, 1347-1364. [CrossRef]

19. Park, B.G.; Kim, T.H.; Tahk, M.J. Range-to-go weighted optimal guidance with impact angle constraint and seeker's look angle limits. IEEE Trans. Aerosp. Electron. Syst. 2016, 52, 1241-1256. [CrossRef]

20. Ratnoo, A. Analysis of two-stage proportional navigation with heading constraints. J. Guid. Control Dyn. 2016, 39, 156-164. [CrossRef]

21. Erer, K.S.; Tekin, R. Impact time and angle control based on constrained optimal solutions. J. Guid. Control Dyn. 2016, 39, 2448-2454. [CrossRef]

22. Kim, H.G.; Lee, J.Y.; Kim, H.J. Look Angle Constrained Impact Angle Control Guidance Law for Homing Missiles With Bearings-Only Measurements. IEEE Trans. Aerosp. Electron. Syst. 2018, 54, 3096-3107. [CrossRef]

23. Sharma, Y.R.; Ratnoo, A. A bearings-only trajectory shaping guidance law with look-angle constraint. IEEE Trans. Aerosp. Electron. Syst. 2019, 55, 3303-3315. [CrossRef]

24. Liu, B.; Hou, M.; Li, Y. Field-of-view and impact angle constrained guidance law for missiles with time-varying velocities. IEEE Access 2019, 7, 61717-61727. [CrossRef]

25. Liu, B.; Hou, M.; Feng, D. Nonlinear mapping based impact angle control guidance with seeker's field-of-view constraint. Aerosp. Sci. Technol. 2019, 86, 724-736. [CrossRef]

26. Han, T.; Hu, Q.; Xin, M. Analytical solution of field-of-view limited guidance with constrained impact and capturability analysis Aerosp. Sci. Technol. 2020, 97, 105586. [CrossRef]

27. Kim, J.; Cho, N.; Kim, Y. Field-of-view-constrained impact angle control guidance with error convergence before interception considering speed changes. Proc. Inst. Mech. Eng. Part G J. Aerosp. Eng. 2021, 235, 238-256. [CrossRef]

28. Jeon, I.S.; Lee, J.I.; Tahk, M.J. Impact-time-control guidance with generalized proportional navigation based on nonlinear formulation. J. Guid. Control Dyn. 2016, 39, 1885-1890. [CrossRef]

29. Jeon, I.S.; Lee, J.I. Impact-time-control guidance law with constraints on seeker look angle. IEEE Trans. Aerosp. Electron. Syst. 2017, 53, 2621-2627. [CrossRef]

30. Kee, P.E.; Dong, L.; Siong, C.J. Near optimal midcourse guidance law for flight vehicle. In Proceedings of the 36th AIAA, Aerospace Sciences Meeting \& Exhibit, Reno, NV, USA, 12-15 January 1998. 